\title{
Guitar Pickup: Measurement of its Two-dimensional Nonlinear Function
}

\author{
Antonin Novak*, Bertrand Lihoreau, Emmanuel Brasseur, \\ Pierrick Lotton, Laurent Simon \\ Laboratoire d'Acoustique de I'Université du Mans, LAUM - UMR 6613 CNRS, Le Mans Université, Avenue Olivier \\ Messiaen, 72085 LE MANS CEDEX 9, France \\ *antonin.novak@univ-lemans.fr, ${ }^{*}$ https ://ant-novak.com
}

\begin{abstract}
The electromagnetic pickup of an electric guitar is a nonlinear device that provides a pleasant distortion. Although the pickup is a simple device consisting of a coil and a few magnets or pole pieces, the measurement of its nonlinear function is a difficult task. This paper shows a measurement technique that can estimate the nonlinear function of a pickup in both $y$ - and $z$ - directions of the vibrating string. The experimental results are provided for three different types of pickups: a single-coil pickup with six staggered pole-pieces, a humbucker pickup with six equal height polepieces, and a humbucker rail pickup. The measured non-linear functions of the three pick-ups are very different from each other, leading to different distortions. These experimental results confirm that the pickup geometry plays an important role in distortion.
\end{abstract}

The archived file is not the final published version of the article $* * * * * * * * *$

The definitive publisher-authenticated version is available online at $* * * * *$, Readers must contact the publisher for reprint or permission to use the material in any form. 


\section{INTRODUCTION}

An electric guitar is one of many musical instruments whose beautiful sound is born with the help of a non-linear mechanism. The heart of an electric guitar is a pickup, a non-linear sensor that picks up the mechanical vibrations of the strings and converts them into an electrical signal $[1,2,3,4,5,6]$. The most common type of pickup is the magnetic one, which houses a coil wrapped around a core composed of either a row of six polar pieces (see Figure 1(a)) or a bar magnet (called rail) (see Figure 1(b)). A ferromagnetic string that vibrates near the pickup causes a variation in the magnetic flux through the coil and, according to Faraday's law, an electrical voltage is induced at the coil's terminals.

There are many pickups available on the market, each of them providing different outputs, some sound warmer, some cleaner and others more distorted. All the electric-guitar players have probably wondered what distinguishes one particular pickup from another. The answer to this puzzling question is essential not only for guitar players but also for pickup manufactures and for digital audio effects engineers, especially those working with instrument synthesis $[7,8,9]$. A few mathematical models of pickup available in the literature may help to find the answer to this tricky question.

Horton and Moore provide a model of the magnetic field of pickups with pole pieces [3]. Guadagnin et al. present an analytical model of the pickup and its influence on pickup distortion [10]. The work of Jungmann [1] provides a very detailed overview of how pickups work and how their output signals may be affected by their physical properties. Lastly, Paiva et al. provide a block-oriented model combining linear and nonlinear blocks to describe the pickup behavior [11]. Based on these works one can conclude that the sound of a pickup is influenced by three main factors: a) the pickup position which is closely related to the string vibration, b) the pickup impedance together with the input impedance of the device to which the guitar is plugged which form a linear filter, and c) a nonlinear behavior of the pickup. Furthermore, the main core of Paiva's model [11] describing the magneto-electric conversion is based on a static nonlinearity representing the nonlinear relation between the string displacement and the magnetic flux, followed by a time differentiator.

Many of these studies focus on theoretical modeling and simulations. The work presented in this paper uses an experimental approach and tries to answer the following questions from the experimental results. What distinguishes one pickup from other from the nonlinear distortion point of view? Can the distortion contribution due to the horizontal excitation be neglected? Does the distortion depend on the string rest position?

The work presented in this paper is a continuity of the recently published work [12], where a dedicated experimental bench that provides a purely sinusoidal string excitation was used to show the harmonic distortion of several pickups. In [12], the pickups are excited in $z$-direction (Fig. 1) and the static nonlinear function of the pickup is measured using the dedicated bench. In this work, the same experimental bench as the one used in [12] is used to

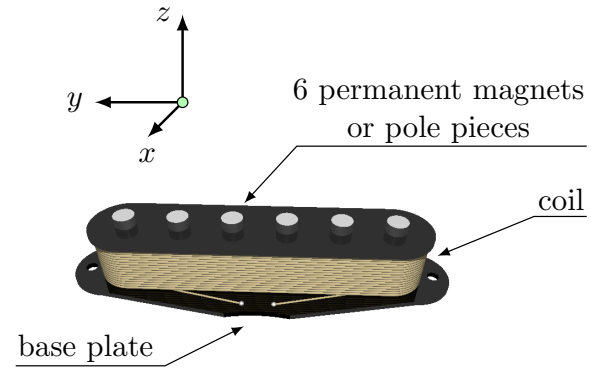

a) pickup with six pole pieces

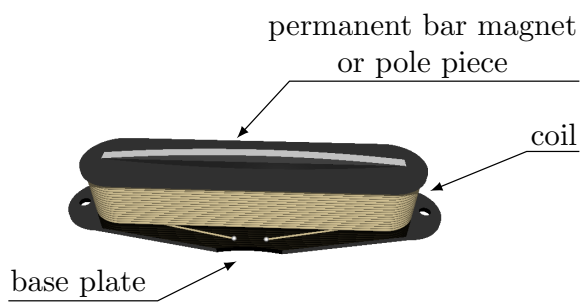

b) rail pickup

Figure 1. Schematic representation of (a) a pole-piece pickup and (b) a rail pickup. 


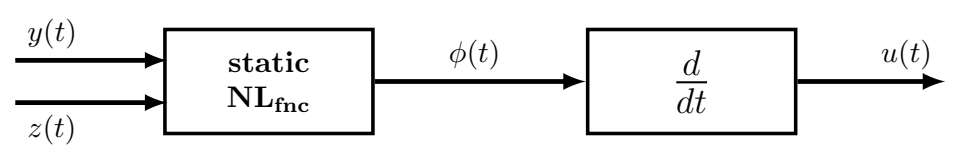

Figure 2. Block-oriented representation of the pickup nonlinear model with both horizontal $y(t)$ and vertical $z(t)$ string displacement input and the voltage output $u(t)$.

measure the static nonlinear function of three different pickups in both vertical $(z-)$ and horizontal $(y$ - $)$ directions. As the nonlinear behavior of the pickup is influenced by the vibration of the string in both directions $(y$ and $z)$, Paiva's static nonlinear model is extended to two inputs (Fig. 2). The experimental results verify some of earlier theoretical results for both vertical $[1,4,3]$ and horizontal $[4,3]$ directions.

\section{EXPERIMENTAL SETUP}

\subsection{Pickups under test}

Three types of pickups of the brand Seymour Duncan are used in this work to measure their differences from the nonlinear point of view: (a) SSL-5 - a single-coil pickup, (b) SH-2N - a humbucker (double-coil) pickup, and (c) STHR-1B - a humbucker rail pickup (Fig. 3).

\subsection{Experimental bench}

A guitar pickup is one of the non-linear systems that are not easy to measure. E.g., a precise measurement of the harmonic distortion at the output of the pickup requires a pure sine motion of the string as the input signal. Since the guitar's string displacement is guided by the laws of vibrations, the input signal of the pickup is difficult to control.

To impose the string displacement we use a dedicated measurement bench $[12,13]$ whose schematic representation is depicted in Fig. 4 for vertical ( $z$-axis) motion of the string. A piece of string ( $8 \mathrm{~cm}$ long and $1 \mathrm{~mm}$ in diameter) is glued to a composite plate $(3 \times 8 \mathrm{~cm})$ which is rigidly connected to an electrodynamic shaker (LDS V406). The shaker, driven by a Devialet D-premier amplifier, RME Fireface 400 sound card, and a personal computer, is used as a source of the string displacement. The pickup is then placed to the proximity of the string so that the string rest position is at coordinates $\left(y_{0}, z_{0}\right)$. An electromagnetic shielding cage is placed around the shaker to avoid a possible disturbance by the electromagnetic field of the shaker. An accelerometer PCB $352 \mathrm{C} 22$ is fixed to the composite plate and is connected to a PCB sensor signal conditioner 482C series. The sound card RME Fireface 400 is then used to acquire both the accelerometer signal $a(t)$ and the output voltage $u(t)$ from the pickup (directly connected to the sound card instrument input with an impedance of $470 \mathrm{k} \Omega$ ).

To ensure that the string is vibrating in a pure sinusoidal way with no higher harmonic components due to the nonlinear behavior of the shaker we use a recently developed adaptive technique of harmonic control that can

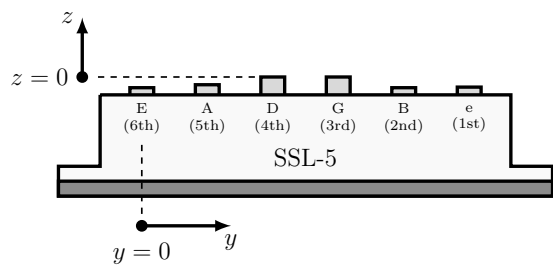

a) SSL-5 pickup

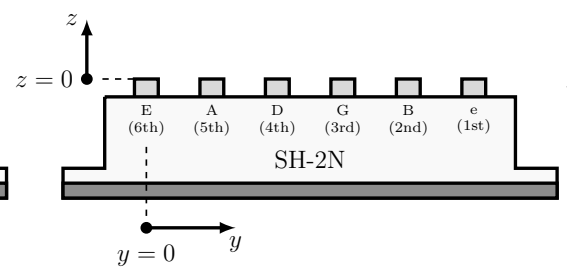

b) $\mathrm{SH}-2 \mathrm{~N}$ pickup

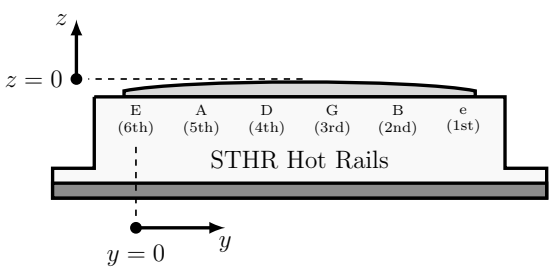

c) STHR pickup

Figure 3. Profiles of three pickups under test. 


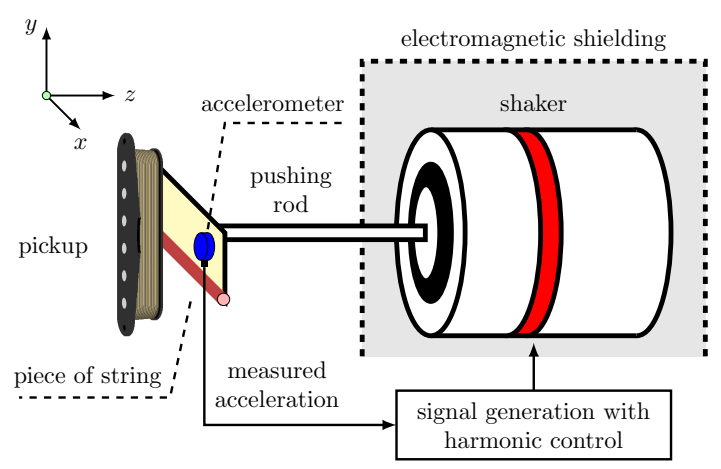

a) experimental setup for vertical excitation

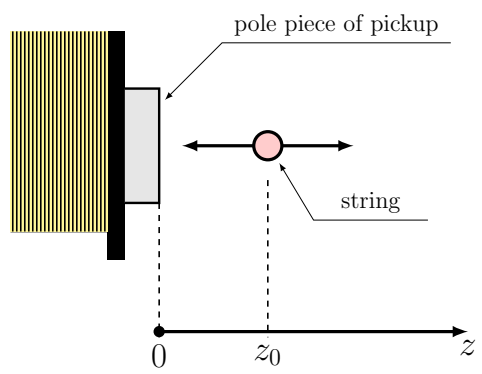

b) string position (vertical excitation)

Figure 4. Schematic representation of the measurement for the vertical ( $z$-direction) string motion.

pre-distort the input signal of the shaker to create a perfect periodical signal at the output of the shaker with spectral purity up to $100 \mathrm{~dB}$ [14]. Using this technique, we can generate a pure harmonic displacement of the string even for large amplitudes, completely getting rid of the higher harmonics created by the shaker.

To enable the measurement not only in vertical $z$-direction but also in horizontal $y$-direction the measurement setup is modified as depicted in Fig. 5(a). The pickup is placed horizontally, and the $y$-axis is oriented in accordance with Fig. 5(b). The distance between the string at rest and the pole piece is still denoted $z_{0}$.

\subsection{Harmonic distortion}

An example of harmonic distortion of each pickup is shown in Fig. 6 for both vertical (along $z$-axis) and horizontal (along $y$-axis) string displacements. The rest position of the string is set at the distance of $z_{0}=3 \mathrm{~mm}$ in front of the fifth string position $\left(y_{0}=9.5 \mathrm{~mm}\right)$. The string is then displaced with a pure sinusoidal excitation of frequency $110 \mathrm{~Hz}$ (corresponding to open A-string) and amplitude of $\pm 1.5 \mathrm{~mm}$ in (a) vertical excitation and (b) horizontal excitation. The spectra of the voltage signal at the output of each pickup are depicted in Fig. 6 .

One can note that the vertical excitation leads to a voltage signal about $20 \mathrm{~dB}$ higher than the horizontal excitation. On the one hand, the vertical excitation leads to a generation of similar spectra for all three tested pickups; the STHR-1B rail pickup providing slightly stronger distortion compared to the other two pickups. On the other hand, the horizontal excitation shows completely different spectra for all three pickups. The SSL-5 pickup excited horizontally generates the first and the second harmonic at the level of $-28 \mathrm{~dB}$ and $-35 \mathrm{~dB}$ respectively. The dominant harmonic of the SH-2N pickup is the second one $(-29 \mathrm{~dB})$; the first harmonic is attenuated to $-53 \mathrm{~dB}$. The STHR-1B rail pickup has the first harmonic the strongest $(-29 \mathrm{~dB})$, the other harmonic being much smaller $(-50 \mathrm{~dB}$ for the second harmonic). 


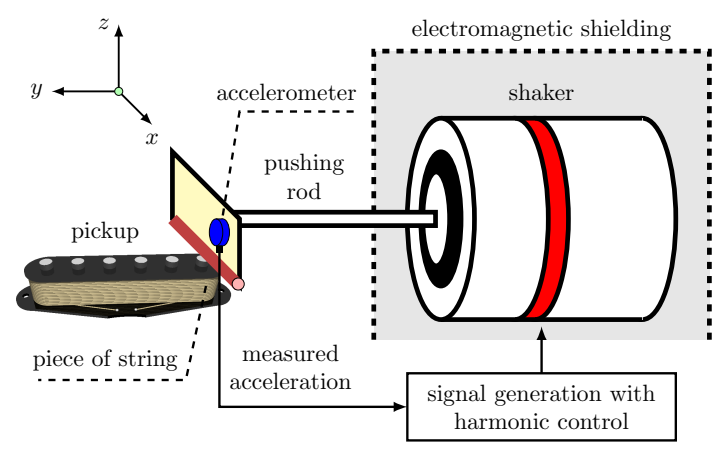

a) experimental setup for horizontal excitation

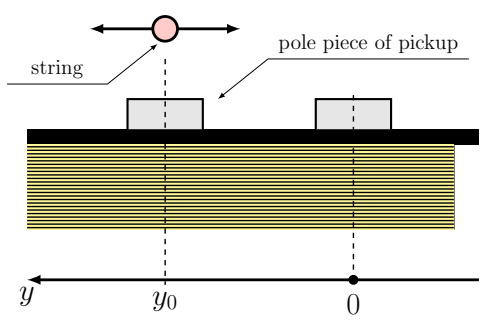

b) string position (horizontal excitation)

Figure 5. Schematic representation of the measurement for the horizontal (in $y$-direction) string motion.

The following experimental study is focused on the measurement of the static nonlinear function $\mathrm{NL}_{\mathrm{fnc}}$ and its influence on the nonlinear distortion of each pickup under test.

\section{STATIC NONLINEAR FUNCTION}

The block-oriented model of the pickup proposed by Paiva [11] (static nonlinearity $\mathrm{NL}_{\mathrm{fnc}}$ followed by the time derivative) has been experimentally verified in several works using the vertical excitation [15, 16, 12]. In [12] it has been shown that the static nonlinear function $\mathrm{NL}_{\mathrm{fnc}}$ can be obtained experimentally using the following procedure.

First, we can deduce the variable $\phi(t)$ from Paiva's model using Faraday's law of induction

$$
u(t)=-\frac{d \phi(t)}{d t}
$$

that defines the voltage $u(t)$ generated at the output of a coil. Next, we obtain $\phi(t)$ as an integral of the measured voltage $u(t)$

$$
\phi(t)=-\int_{-\infty}^{t} u\left(t^{\prime}\right) d t^{\prime}+C,
$$

where an unknown constant of integration $C$ is inherent in the construction of anti-derivatives. Finally, the static nonlinear function $\mathrm{NL}_{\text {fnc }}$ is plotted directly from measured displacement $z(t)$ (deduced from the acceleration) and the time integral of the voltage as plot $(z, p h i)$ in Matlab language. This procedure is graphically represented in Fig. 7 with measured data provided from $60 \mathrm{~Hz}^{1}$ excitation with a sinusoidal $z$-axis displacement of $\pm 3 \mathrm{~mm}$ around $y_{0}=9.5 \mathrm{~mm}$ and $z_{0}=3 \mathrm{~mm}$.

\footnotetext{
${ }^{1}$ Note that $60 \mathrm{~Hz}$ is the resonant frequency of the shaker at which it can reach high displacement $( \pm 3 \mathrm{~mm})$.
} 


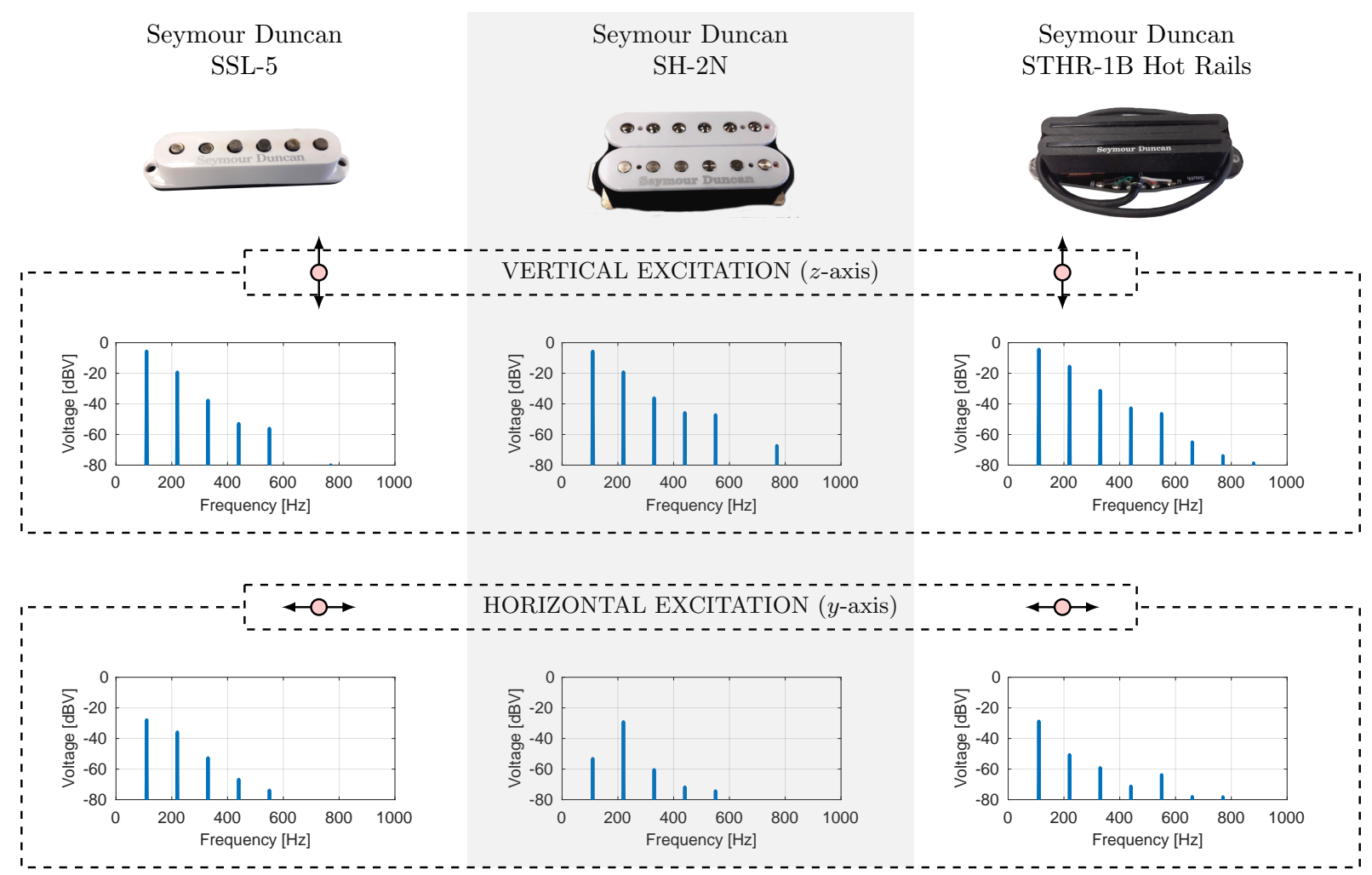

Figure 6. Spectra of the output voltage of three pickups under test obtained with the excitation frequency of 110 Hz: a single-coil pickup with six staggered pole-pieces on the left, a humbucker pickup with six equal height pole-pieces in the middle, and a humbucker rail pickup on the right. Results depicted for both vertical and horizontal string motions. The string is placed at the distance of $z_{0}=3 \mathrm{~mm}$ in front of the fifth pole piece position $\left(y_{0}=9.5 \mathrm{~mm}\right)$.

The results from [12] show that the whole nonlinear function $\mathrm{NL}_{\mathrm{fnc}}$ can be built from several measurements for different rest positions of the string $z_{0}$ by adjusting the unknown constant $C$ for each measurement as depicted in Fig. 8. It is also shown in [12] that the results are independent of the excitation frequency, which is in accordance with Paiva's hypothesis of static nonlinear function (static meaning independent of frequency).

The procedure used for the measurement with the vertical ( $z$ - axis) excitation is next used for horizontal $(y-$ axis) excitation. The pickup is placed as depicted in Fig. 5. The string is placed at its rest position $\left(y_{0}, z_{0}\right)$, displaced horizontally along the $y$-axis with the shaker, and the static nonlinear function $\mathrm{NL}_{\mathrm{fnc}}$ is estimated using the same procedure as for the vertical excitation. An example with a $60 \mathrm{~Hz}$ excitation with a sinusoidal $y$-axis displacement of $\pm 3 \mathrm{~mm}$ around $y_{0}=9.5 \mathrm{~mm}$ and $z_{0}=3 \mathrm{~mm}$ is shown in Fig. 9 for the $\mathrm{SH}-2 \mathrm{~N}$ pickup. The same measurement is next made for several values of $y_{0}$, as shown in Fig. 10.

The static nonlinear function $\mathrm{NL}_{\mathrm{fnc}}$ in the horizontal direction $\left(z_{0}=3 \mathrm{~mm}\right)$ for the $\mathrm{SH}-2 \mathrm{~N}$ pickup depicted in Fig. 10 shows an interesting pattern. The nonlinear function $\mathrm{NL}_{\text {fnc }}$ has two local maxima at $y_{0}=0 \mathrm{~mm}$ and $y_{0}=9.5 \mathrm{~mm}$ corresponding to the 6 th and 5 th pole pieces of the pickup. The $\mathrm{NL}_{\text {fnc }}$ also has two local minima in-between the positions of pole pieces. Finally, the $\mathrm{NL}_{\mathrm{fnc}}$ drops for negative values of $y$ (outside the pickup). The relation between the shape of the $\mathrm{NL}_{\mathrm{fnc}}$ and the nonlinear distortion is detailed in section 5 . 


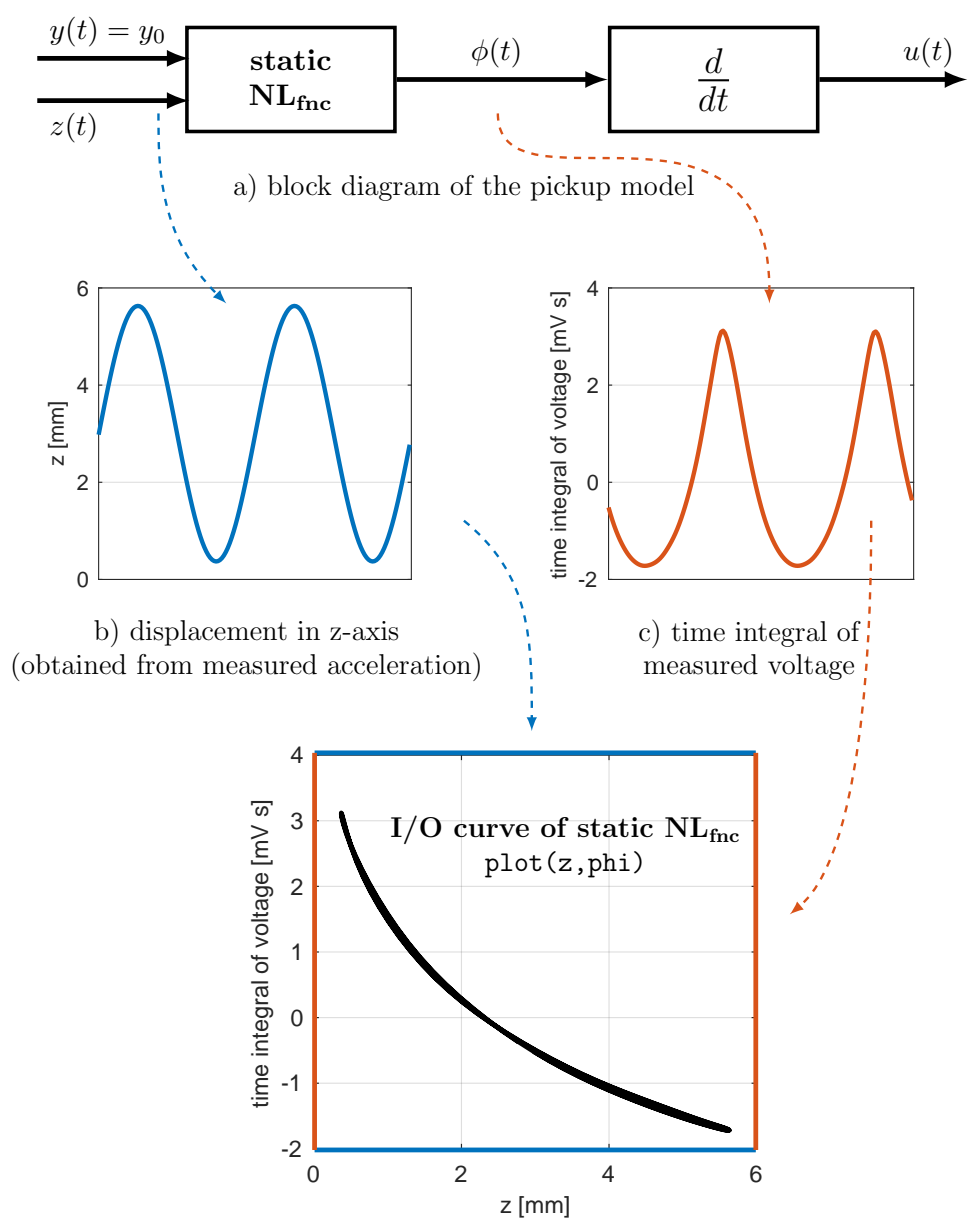

d) time integral of voltage vs. displacement

Figure 7. (a) A block diagram of the pickup nonlinear model, (b) string displacement in vertical direction (obtained from the measured acceleration), (c) time integral of the measured voltage, and (d) a plot of time integral of voltage vs. string displacement in an I/O graph. Measurements performed on a SH-2N pickup with a string rest position $\left(y_{0}=9.5, z_{0}=3\right) \mathrm{mm}$ and $60 \mathrm{~Hz}$ harmonic string oscillation in $z$-direction with amplitude of $\pm 3 \mathrm{~mm}$.

\section{TWO-DIMENSIONAL NONLINEAR FUNCTION}

Both vertical (along $z$-axis) and horizontal (along $y$-axis) measurements for different $\left(y_{0}, z_{0}\right)$ can be joined together to form a two-dimensional function $\mathrm{NL}_{\mathrm{fnc}}(y, z)$ whose value depends on the instantaneous position of the string. Fig. 11 shows the function $\mathrm{NL}_{\text {fnc }}(y, z)$ of the $\mathrm{SH}-2 \mathrm{~N}$ pickup. The function is represented as a two-dimensional graph composed of 44 measured curves (each plotted in a different color). The vertical measurements are provided at a grid of $6 \times 4$ positions $\left(y_{0}, z_{0}\right)$ with $y_{0}$ from -4.75 to $19 \mathrm{~mm}$ with a step of $4.75 \mathrm{~mm}$, and $z_{0} \in(3,5,7$, and $10 \mathrm{~mm})$. The horizontal measurements are provided at a grid of $2 \mathrm{x} 10$ points $\left(y_{0}, z_{0}\right)$ with $y_{0}$ from -8.5 to $18.5 \mathrm{~mm}$ with a step of $3 \mathrm{~mm}$ and $z_{0}$ at 3 and $5 \mathrm{~mm}$.

The flux $\phi(t)$ is given as

$$
\phi(t)=\mathrm{NL}_{\mathrm{fnc}}(y(t), z(t)),
$$

whose time derivative is equal to the phase inverted (minus sign) output voltage of the pickup (see Eq. (1))

$$
u(t)=-\frac{d \mathrm{NL}_{\mathrm{fnc}}(y(t), z(t))}{d t} .
$$




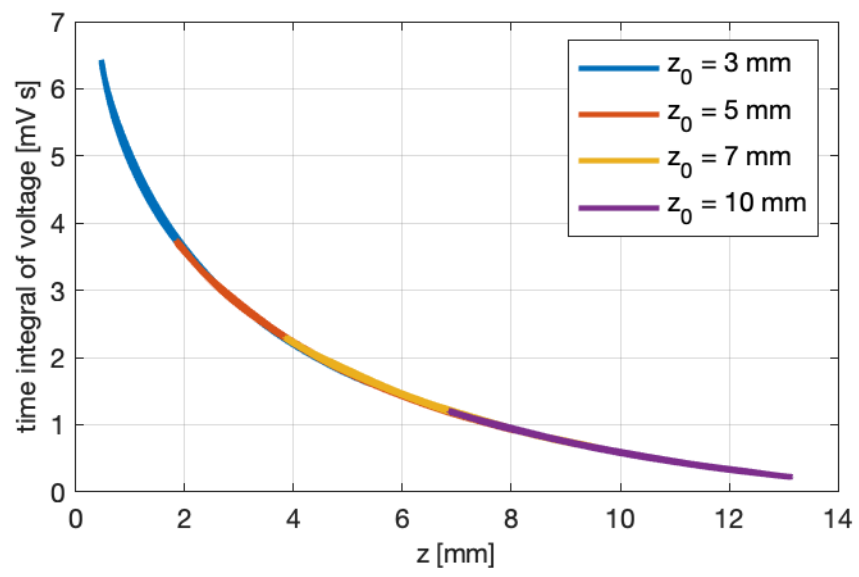

Figure 8. I/O graphs (time integral of measured voltage vs. string displacement in vertical direction) for four different pickup/string distances $z_{0}$. All the four I/O graphs are superposed and shifted by the unknown constant of integration (Eq. (2)). Measurements performed on a SH-2N pickup with a string rest position $\left(y_{0}=9.5 \mathrm{~mm}, z_{0}\right)$ and $60 \mathrm{~Hz}$ harmonic string oscillation in $z$-direction with amplitude of $\pm 3 \mathrm{~mm}$.

Applying the chain rule for the derivative of functions of two variables we can express the output voltage as

$$
u(t)=-\frac{\partial \mathrm{NL}_{\mathrm{fnc}}}{\partial y} \frac{d y(t)}{d t}-\frac{\partial \mathrm{NL}_{\mathrm{fnc}}}{\partial z} \frac{d z(t)}{d t} .
$$

Denoting the velocity vector $\vec{v}(t)$

$$
\vec{v}(t)=\left(\frac{d y(t)}{d t}, \frac{d z(t)}{d t}\right)
$$

and the gradient

$$
\vec{\nabla} \mathrm{NL}_{\mathrm{fnc}}=\left(\frac{\partial \mathrm{NL}_{\mathrm{fnc}}}{\partial y}, \frac{\partial \mathrm{NL}_{\mathrm{fnc}}}{\partial z}\right)
$$

we can express the voltage at the output of the pickup as the following dot product

$$
u(t)=-\vec{\nabla} \mathrm{NL}_{\mathrm{fnc}} \cdot \vec{v}(t) .
$$

Consequently, the distortion of each pickup thus depends on the local gradient of the nonlinear function $\mathrm{NL}_{\mathrm{fnc}}$.

\section{DISCUSSION}

Eq. (8) shows that the pickup output voltage is proportional both to the velocity of the string and to the local gradient of the static nonlinear function $\mathrm{NL}_{\mathrm{fnc}}$. It is thus straightforward to guess from the shape of the function $\mathrm{NL}_{\text {fnc }}$ how the string position $\left(y_{0}, z_{0}\right)$ influences the signal level and the nonlinear distortion of the output voltage.

Fig. 12 shows the results of several vertical (along $z$-axis) and horizontal (along $y$-axis) measurements. The vertical (along $z$-axis) results show that the $\mathrm{NL}_{\mathrm{fnc}}$ decreases with the distance $z$ for all measured pickups but that the gradient (slope) of each function varies with distance $z$. Based on the previous analysis we can conclude that for string positions close to the pickup (e.g., $z_{0}=3 \mathrm{~mm}$ ), in the case of the SSL-5 pickup the $\mathrm{NL}_{\text {fnc }}$ shows lower gradient variation compared to the STHR-1B pickup. This is correlated with the distortion results presented in Fig. 6 where the SSL-5 pickup exhibits lower distortion than the STHR-1B pickup.

The horizontal (along $y$-axis) results presented in Fig. 12 are shown for two different string distances: $z_{0}=3 \mathrm{~mm}$ and $z_{0}=5 \mathrm{~mm}$. Let us first discuss the results for string distances of $z_{0}=3 \mathrm{~mm}$. There is an obvious difference 


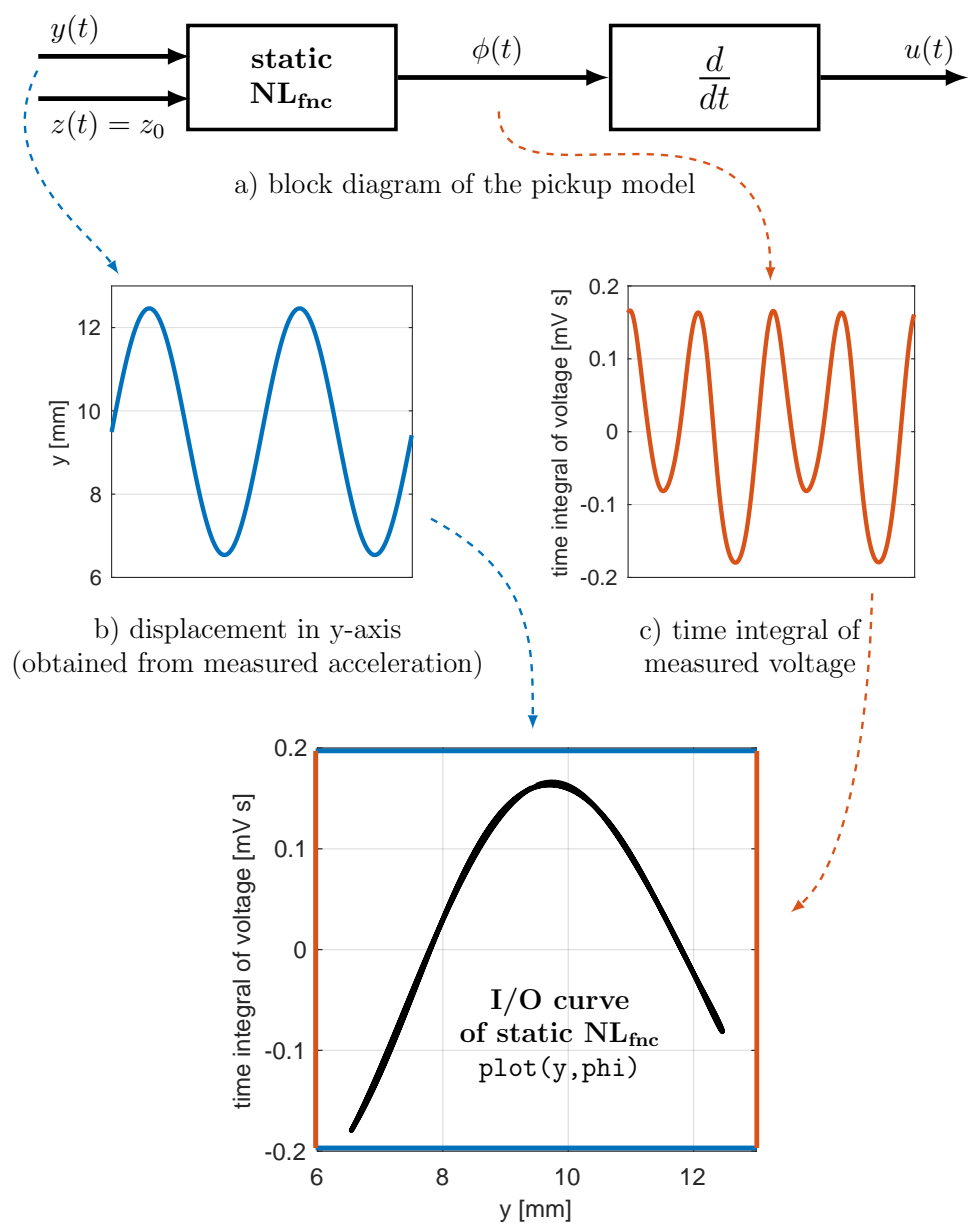

d) time integral of voltage vs. displacement

Figure 9. (a) A block diagram of the pickup nonlinear model, (b) string displacement in horizontal direction (obtained from the measured acceleration), (c) time integral of the measured voltage, and (d) a plot of time integral of voltage vs. string displacement in an I/O graph. Measurements performed on a SH-2N pickup with a string rest position $\left(y_{0}=9.5, z_{0}=3\right) \mathrm{mm}$ and $60 \mathrm{~Hz}$ harmonic string oscillation $y$-direction with amplitude of $\pm 3 \mathrm{~mm}$.

in behavior between the rail pickup (STHR-1B, Fig. 12 right column) and the pickups with pole pieces (SSL-5 and SH-2N, Fig. 12 left and middle column respectively). While there is an obvious influence of pole pieces in the case of SSL-5 and SH-2N resulting in local maxima and minima along the $y$-axis (Fig. 12 left and middle column), the $\mathrm{NL}_{\text {fnc }}$ of the STHR-1B pickup is smooth with no oscillations (Fig. 12, right column). The local maxima and minima of the $\mathrm{NL}_{\mathrm{fnc}}$ of the pole-piece pickups (SSL-5 and SH-2N) are located at positions $y_{0}=0, y_{0}=9.5 \mathrm{~mm}$, and $y_{0}=19 \mathrm{~mm}$ corresponding to positions of $6 \mathrm{th}, 5 \mathrm{th}$, and $4 \mathrm{th}$ pole piece respectively. Measurements in the horizontal direction at different string positions $z_{0}=3 \mathrm{~mm}$ and $z_{0}=5 \mathrm{~mm}$ (Fig. 12) show that for higher $z_{0}$ (string positions further away from pickup) the variations of function $\mathrm{NL}_{\mathrm{fnc}}$ are much smaller.

The shape of the function $\mathrm{NL}_{\text {fnc }}$ in the horizontal direction (Fig. 12) also has an influence on the nonlinear distortion shown in Fig. 6. In Fig. 6, the harmonic distortion is evaluated for the position $\left(y_{0}=9.5 \mathrm{~mm}, z_{0}=3 \mathrm{~mm}\right)$. Fig. 12 shows that at this position for horizontal excitation there is a local maximum in the case of SSL-5 and SH-2N. In the close proximity of this maximum the function $\mathrm{NL}_{\text {fnc }}$ behaves rather symmetrically. The symmetry is almost 


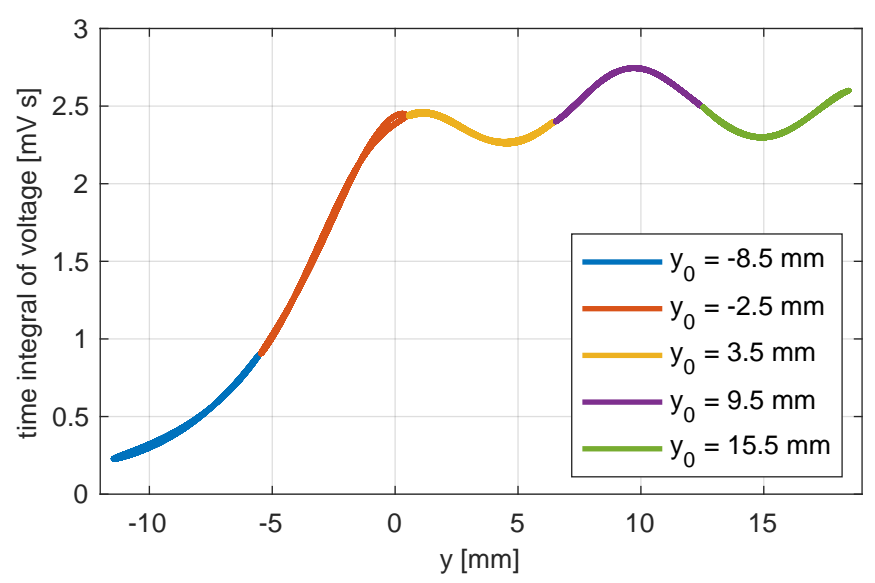

Figure 10. I/O graphs (time integral of measured voltage vs. string displacement in horizontal direction) for five different pickup/string distances $y_{0}$. All the five I/O graphs are superposed and shifted by the unknown constant of integration (Eq. (2)). Measurements performed on a SH-2N pickup with a string rest position $\left(y_{0}, z_{0}=3 \mathrm{~mm}\right)$ and $60 \mathrm{~Hz}$ harmonic string oscillation in $y$-direction with amplitude of almost $\pm 3 \mathrm{~mm}$.

perfect for the SH-2N pickup which is well correlated with the dominating 2nd harmonic (see Fig. 6). For the SSL-5 pickup the symmetry is not perfect resulting in a presence of both 1st and 2nd harmonic components (see Fig. 6). Finally, the STHR-1B pickup shows a smooth nonlinear function $\mathrm{NL}_{\mathrm{fnc}}$ (Fig. 12) which is due to the rail magnet (single bar pole piece). Consequently, the distortion of the STHR-1B pickup in the horizontal direction generates mainly the 1st harmonic (see Fig. 6).

The shape of the function $\mathrm{NL}_{\text {fnc }}$ in horizontal direction (Fig. 12) also indicates how the $y_{0}$ position influences the level and the distortion of the pickup voltage signal. In the case of the STHR-1B rail pickup (Fig. 12, right column, horizontal excitation), the function is almost flat around 4 th string position $\left(y_{0}=19 \mathrm{~mm}\right)$. Its gradient is almost zero leading to almost no voltage output for horizontal excitation. On the other hand, the gradient around 6 th position $\left(y_{0}=0\right)$ is higher, leading to a higher voltage output.

Similar behavior can be observed in the case of SSL-5 and SH-2N pickups (Fig. 12, left and middle column for horizontal excitation): the voltage level and distortion change with the position $y_{0}$. We can also observe a different behavior in the horizontal direction between SSL-5 and SH-2N pickups. Indeed, the SH-2N pickup pole pieces have all the same height while the SSL-5 pickup have the pole pieces staggered (the height of each pole piece in $z$-direction is different), see Fig. 3. The staggered pickup thus breaks the symmetry of the function $\mathrm{NL}_{\mathrm{fnc}}$ (Fig. 12, left column, horizontal excitation).

Although horizontal excitation generates lower output voltage than vertical excitation, it seems important to take this into account for modeling higher harmonics. Distortion measurement of SH-2N pickup (Fig. 6) shows that horizontal excitation can generate a second harmonic whose amplitude is higher than the third harmonic caused by vertical excitation and which is $25 \mathrm{~dB}$ below the first harmonic. A more detailed psycho-acoustic study is needed to draw conclusions about the audibility of these non-linear effects of guitar pick-ups.

\section{CONCLUSION}

Why do some guitar pickups distort more than others? This paper provides a part of the answer to this puzzling question by showing how to measure the static nonlinear function of guitar pickup as a function of the vertical and horizontal displacements of the string. The estimation of the non-linear function can be very useful for the design of pickups. 


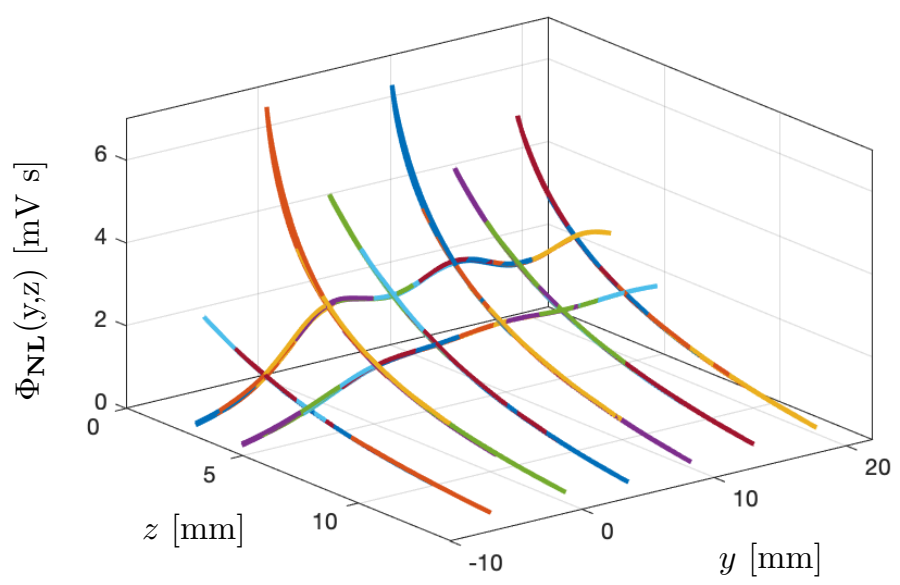

Figure 11. Nonlinear function of the SH-2N pickup obtained as a superposition of all the measured I/O curves and depicted in a single three-dimensional graph.

Three different pickups, a single-coil pickup with six staggered pole-pieces, a humbucker pickup with six equal height pole-pieces, and a humbucker rail pickup, are measured. It is shown that the gradient of the nonlinear static function is directly related to pickup distortion and that vertical and horizontal string vibrations contribute to pickup distortion.

The results show that all three studied pickups have a similar shape of the nonlinear function for the vertical displacement of the string. However, the gradient of this function and its amplitude, both responsible for output level and distortion, differ from one pickup to another. The results also show that the nonlinear function for horizontal string displacement is highly dependent on the geometry of the polar pieces of the pickup. For the rail pickup (single pole pieces), the nonlinear function is flat and smooth, resulting in very low distortion due to the horizontal displacement of the strings. For pickups with six pole pieces, the nonlinear function consists of small local minima and maxima, which leads to a much higher nonlinear distortion for horizontal excitation. While the horizontal excitation provides smaller output voltage than the vertical one, its nonlinear contribution to the pickup distortion can not be neglected, especially for pickups with six pole pieces.

\section{ACKNOWLEDGMENT}

The measurements, discussions, and redaction of this paper have been conducted mainly in a free time of all the authors, motivated by their passion for guitars and nonlinear systems. We would very much like to thank our wives and families for their understanding. 
Seymour Duncan

SSL-5

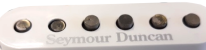

- 0 웅
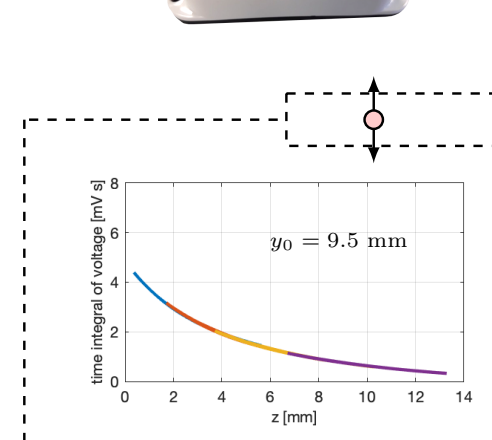

Seymour Duncan SH-2N
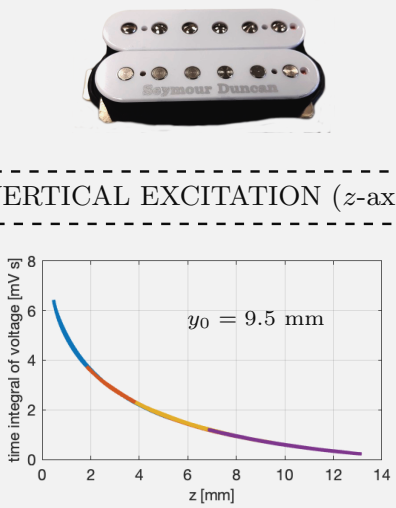

Seymour Duncan STHR-1B Hot Rails

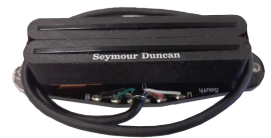

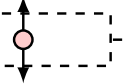

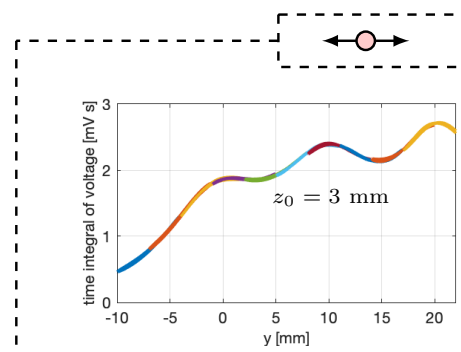

HORIZONTAL EXCITATION ( $y$-axis)
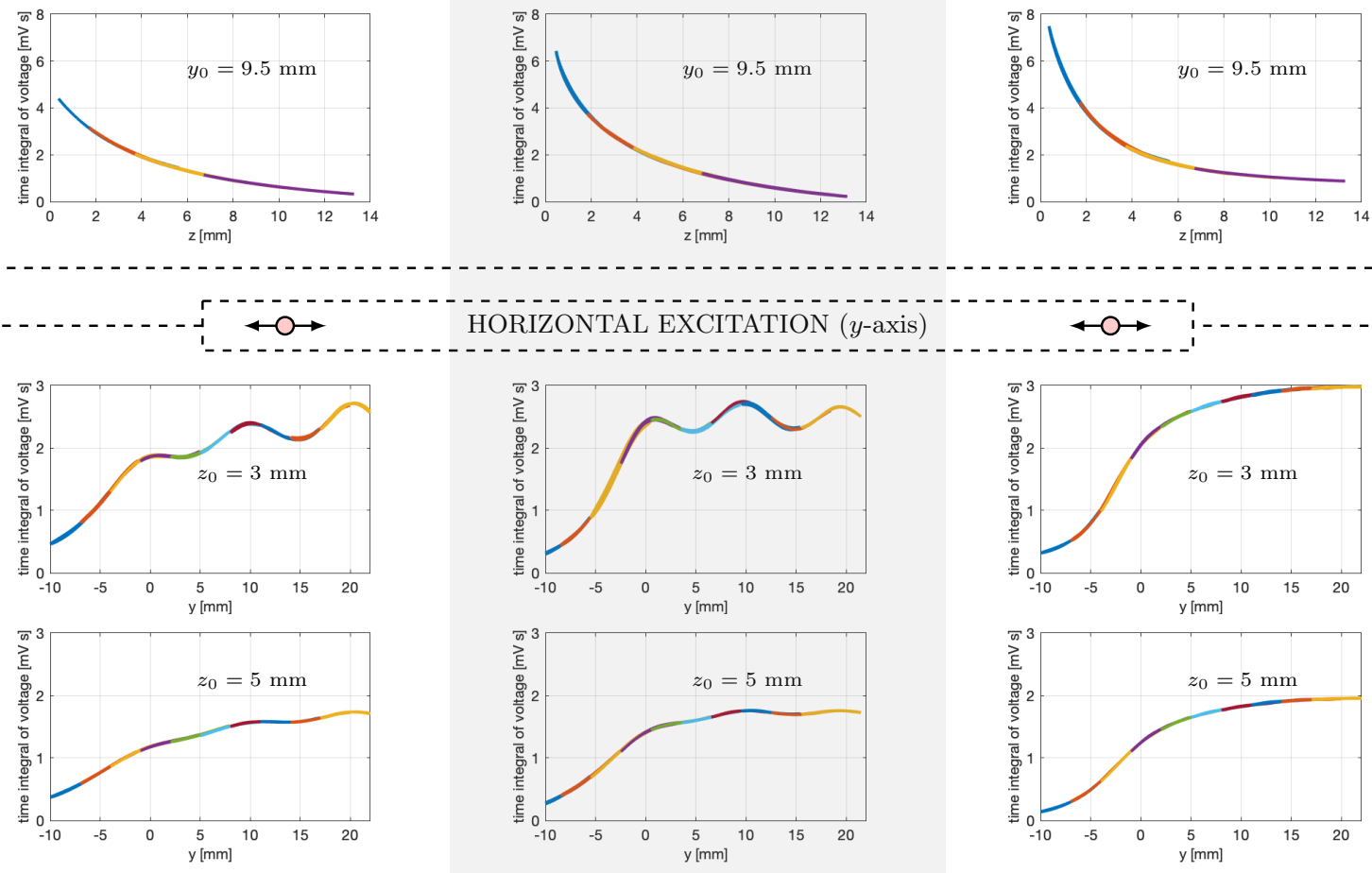

Figure 12. Comparison of the measured nonlinear functions for the three tested pickups depicted for one vertical excitation around $y_{0}=9.5 \mathrm{~mm}$ and two horizontal excitations with $z_{0}=3 \mathrm{~mm}$ and $y_{0}=5 \mathrm{~mm}$. 


\section{References}

[1] T. Jungmann, Theoretical and practical studies on the behavior of electric guitar pick-ups, Master's thesis, Helsinki Univ. of Tech., Espoo, Finland (1994).

[2] D. Hunter, The Guitar Pickup Handbook: The Start of Your Sound (Hal Léonard Corporation) (2008).

[3] N. G. Horton, T. R. Moore, "Modeling the magnetic pickup of an electric guitar," American Journal of Physics, vol. 77 , no. 2, pp. 144-150 (2009).

[4] M. Zollner, Physik der Elektrogitarre (im Selbstverlag, manfred.zollner@oth-regensburg.de) (2014).

[5] M. Mustonen, D. Kartofelev, A. Stulov, V. Välimäki, "Experimental verification of pickup nonlinearity," presented at the Proceedings International Symposium on Musical Acoustics (ISMA 2014), Le Mans, France, vol. 1 (2014).

[6] A. Falaize, T. Hélie, "Guaranteed-passive simulation of an electro-mechanical piano: A port-Hamiltonian approach," presented at the Proc. of the 18 th Int. Conference on Digital Audio Effects (DAFx-15) (2015).

[7] V. Välimäki, J. Huopaniemi, M. Karjalainen, Z. Jánosy, "Physical Modeling of Plucked String Instruments with Application to Real-Time Sound Synthesis," J. Audio Eng. Soc, vol. 44, no. 5, pp. 331-353 (1996).

[8] M. Karjalainen, H. Penttinen, V. Välimäki, "Acoustic sound from the electric guitar using DSP techniques," presented at the Acoustics, Speech, and Signal Processing, 2000. ICASSP'00. Proceedings. 2000 IEEE International Conference on, vol. 2, pp. II773-II776 (2000).

[9] M. Karjalainen, T. Mäki-Patola, A. Kanerva, A. Huovilainen, "Virtual air guitar," Journal of the Audio Engineering Society, vol. 54, no. 10, pp. 964-980 (2006).

[10] L. Guadagnin, B. Lihoreau, P. Lotton, E. Brasseur, "Analytical Modeling and Experimental Characterization of a Magnetic Pickup for Electric Guitar," Journal of the Audio Engineering Society, vol. 65, no. 9, pp. 711-721 (2017).

[11] R. C. Paiva, J. Pakarinen, V. Välimäki, "Acoustics and modeling of pickups," Journal of the Audio Engineering Society, vol. 60, no. 10, pp. 768-782 (2012).

[12] A. Novak, B. Lihoreau, P. Lotton, E. Brasseur, L. Simon, "Experimental Study Of Guitar Pickup Nonlinearity," presented at the Proc. of the 18th Int. Conference on Digital Audio Effects (DAFx-18) (2018).

[13] P. Lotton, B. Lihoreau, E. Brasseur, "Experimental Study of a Guitar Pickup," presented at the Int. Symp. Music. Acoust., pp. 355-360 (2014).

[14] A. Novak, L. Simon, P. Lotton, "A simple predistortion technique for suppression of nonlinear effects in periodic signals generated by nonlinear transducers," Journal of Sound and Vibration, vol. 420, pp. 104-113 (2018).

[15] A. Novak, L. Guadagnin, B. Lihoreau, P. Lotton, E. Brasseur, L. Simon, "Non-linear identification of an electric guitar pickup," presented at the Proceedings of the 19th International Conference on Digital Audio Effects (DAFx-16), Brno, Czech Republic, pp. 5-9 (2016).

[16] A. Novak, L. Guadagnin, B. Lihoreau, P. Lotton, E. Brasseur, L. Simon, "Measurements and Modeling of the Nonlinear Behavior of a Guitar Pickup at Low Frequencies," Applied Sciences, vol. 7, no. 1, p. 50 (2017). 